\title{
Psychosocial Distress in Follow-up Care - Results of a Tablet-based Routine Screening in 202 Patients With Sarcoma
}

\author{
FLORIAN LENZE ${ }^{1}$, FLORIAN POHLIG ${ }^{1}$, CAROLIN KNEBEL $^{1}$, HEINRICH MÜHLHOFER ${ }^{1}$, HANS RECHL $^{1}$, \\ THERESIA PICHLER ${ }^{2}$, PETER HERSCHBACH ${ }^{3}$, RÜDIGER VON EISENHART-ROTHE $^{1}$ and ULRICH LENZE $^{1}$ \\ ${ }^{1}$ Department of Orthopedics and Sports Orthopedics, Klinikum rechts der Isar, \\ Technical University Munich, Munich, Germany; \\ ${ }^{2}$ CCC Comprehensive Cancer Center, Munich, Germany; \\ ${ }^{3}$ Roman Herzog Comprehensive Cancer Center, Department of Psychosomatic Medicine and Psychotherapy, \\ Klinikum rechts der Isar, Technical University Munich, Munich, Germany
}

\begin{abstract}
Background: Patients with sarcoma are particularly vulnerable to psychosocial distress. The aim of this study was to collect preliminary data on the prevalence of psychosocial distress in such patients during follow-up care and identify risk factors associated with higher psychooncological stress levels. Patients and Methods: The study retrospectively enrolled 202 patients with bone or soft-tissue sarcomas who underwent routine psychosocial distress screening during their follow-up care. All patients were screened using an electronic cancer-specific questionnaire. Results: Females and patients who underwent radiotherapy were more distressed. Psychosocial distress levels were markedly higher in the early postoperative phase, but approximately one-third of patients showed high psychosocial distress levels even more than 2 years postoperatively. Conclusion: The results underscore the importance of routine psychosocial distress screenings in patients with sarcoma, which should be performed throughout the follow-up period.
\end{abstract}

Approximately $30-66 \%$ of all patients with cancer suffer from psychosocial distress during the course of their disease, which constitutes a relevant clinical and economical problem (1-4). However, treatment of such patients is still mainly focused on biomedical aspects. Studies on psychosocial distress in patients with cancer have shown that the identification of distressed patients is a considerable challenge $(5,6)$. This difficulty might

Correspondence to: Florian Lenze, MD, Department of Orthopedics and Sports Orthopedic, Klinikum rechts der Isar, Technical University Munich, Ismaninger Str. 22, 81675 Munich, Germany. E-mail: Flo_Lenze@yahoo.de

Key Words: Sarcoma, psychosocial distress, distress screening, cancer, QSC-R10. be attributed to the weak correlation between objective disease characteristics/symptoms and the subjective perception of distress of patients with cancer (5-8). Additionally, many patients feel discomfort when talking about mental health issues, and approximately 25\% speak about their mental concerns only when invited to do so (9). International guidelines suggest routine distress screening for all patients with cancer, and such screening is one of the certification requirements for cancer centres $(7,10)$. In this context, we implemented routine psychosocial distress screenings for all patients with cancer at our Orthopaedic University Department.

Malignant tumours of the musculoskeletal system are rare, comprising only $1 \%$ of all malignant tumours (11). Despite improvements in therapy and increased survival rates, patients with musculoskeletal malignancies, such as softtissue sarcomas, are particularly vulnerable to psychosocial distress (12-15). In our experience, high distress levels occur not only at early stages of the disease but also during the follow-up period (even years after the operation) in some patients.

Most studies on the prevalence of psychosocial distress in patients with extremity sarcomas use common screening instruments such as the Brief Symptom Inventory (BSI), the Hospital Anxiety and Depression Scale (HADS) or the Depression and Anxiety Stress Scale 21 (DASS 21) (14, 1619). These questionnaires, however, are not cancer-specific and mainly assess the prevalence of psychiatric disorders, especially anxiety and depression. In contrast, cancerspecific screening instruments specifically focus on diseaserelated distress and highlight the patient's subjective experiences of cancer. Therefore, such instruments may be of higher clinical value in terms of deriving treatment consequences (12). A popular and validated cancer-specific tool for assessing psychosocial distress is the Questionnaire on Stress in Cancer Patients (QSC-R23), which contains 23 items for describing potential everyday stress in all areas of 
life for such patients (12). For our routine screening, we use the QSC-R10 - a 10-item short form of the QSC-R23, which was developed to improve its applicability in clinical practice. Book et al. demonstrated the high validity and reliability of this instrument in a sample of 1,850 patients with cancer (3). The use of electronic screening systems provides immediate scoring of questionnaire results, facilitates data collection and can help to ensure the overall efficiency of psychosocial screening programmes (20-23). Studies have suggested a high acceptance of computerized screenings even among older patients and those without computer experience $(24,25)$.

The aims of this study were as follows: i) To gather preliminary data on the prevalence of psychosocial distress in sarcoma patients during follow-up care; ii) To identify risk factors that might be associated with higher psychooncological stress levels in patients with orthopaedic cancer.

\section{Patients and Methods}

Participants. We retrospectively enrolled all patients who were screened during their follow-up care for psychosocial distress following resection of a malignant musculoskeletal tumour between 2014 and 2017.

Inclusion criteria comprised follow-up care after resection of a bone or soft-tissue sarcoma of the extremities, as well as an age of 18 years or above. Exclusion criteria were benign musculoskeletal tumours or sarcomas at locations other than the extremities, unresected malignant tumours (that had not been resected at the time of screening), pre-existing psychiatric disorders, insufficient language skills, and an age below 18 years.

The study was approved by the local Ethics Committee (project number 216/11).

Procedure. At our Orthopaedic Department, routine screening is performed in the form of a tablet-based self-assessment using the QSC-R10. To carry out this screening, a screening terminal consisting of a tablet stand and a tablet computer has been set up in a designated area of our Orthopaedic Outpatient Clinic. Upon arrival in the clinic, all patients receive concise instructions on how to handle the tablet computer as well as the questionnaire in order to complete all questions on their own. All patients included in this study underwent an identical psycho-oncological screening procedure independent of disease-related factors such as entity (soft-tissue tumour, bone tumour, etc.), tumour stage (local disease, metastatic, etc.), grading, type of surgery (amputation, limb preservation, etc.), additional treatment (radiotherapy, chemotherapy, etc.), and the length of the postoperative period. If one patient underwent more than one psychosocial distress screening during the study period, only the data from the first screening were included and used for the analysis.

After completion of all questions, data were automatically analysed in terms of current distress level (see below) as well as the subjective desire for psycho-oncological support. Individual result sheets were transferred to the medical file of each patient, and the findings were discussed during the outpatient visit (same day) by the attending orthopaedic doctor.

Patients who exceeded the defined cutoff value were assumed to be highly distressed and were offered psycho-oncological support
Table I. Medical and socio-demographic profile of patients $(n=202)$.

\begin{tabular}{|c|c|}
\hline Characteristic & $\mathrm{n}(\%)$ \\
\hline \multicolumn{2}{|l|}{ Socio-demographic } \\
\hline \multicolumn{2}{|l|}{ Gender, n (\%) } \\
\hline Female & $93(46)$ \\
\hline Male & $109(54)$ \\
\hline \multicolumn{2}{|l|}{ Age at diagnosis (years) } \\
\hline Mean (SD) & $51.9(13.0)$ \\
\hline Min. & 18 \\
\hline Max. & 92 \\
\hline \multicolumn{2}{|l|}{ Medical } \\
\hline \multicolumn{2}{|c|}{ Duration of postoperative illness $(\mathrm{n}, \%)$} \\
\hline \multicolumn{2}{|c|}{6 Months or less } \\
\hline \multicolumn{2}{|l|}{$53(26)$} \\
\hline 7-12 Months & $57(28)$ \\
\hline 13-24 Months & $28(14)$ \\
\hline Over 24 Months & $64(32)$ \\
\hline \multicolumn{2}{|l|}{ Grading $(\mathrm{n}, \%)$} \\
\hline 1 & $66(35)$ \\
\hline 2 & $58(30)$ \\
\hline 3 & $57(30)$ \\
\hline 4 & $9(5)$ \\
\hline \multicolumn{2}{|c|}{ Location of tumour $(\mathrm{n}, \%)$} \\
\hline Upper extremity & $43(21)$ \\
\hline Lower extremity & $138(68)$ \\
\hline Pelvis & $10(5)$ \\
\hline Thoracic wall & $9(4)$ \\
\hline Neck & $2(1)$ \\
\hline \multicolumn{2}{|l|}{ Type of tumour $(\mathrm{n}, \%)$} \\
\hline Bone sarcoma & $63(31)$ \\
\hline Soft-tissue sarcoma & $139(69)$ \\
\hline Metastasis & $27(13)$ \\
\hline \multicolumn{2}{|l|}{ Active treatment } \\
\hline Chemotherapy & $31(15)$ \\
\hline Radiotherapy & $94(47)$ \\
\hline
\end{tabular}

or intensified treatment (if already receiving psycho-oncological treatment). Likewise, patients who expressed a desire for psychooncological support were referred to the Department of Psychosomatic Medicine and Psychotherapy. For most patients, first contact was made on the same day (day of referral), and further treatment sessions were performed depending on the individual needs of each patient. The psycho-oncological treatment included talking therapy as well as instructions for stress-relief strategies.

Measures. The QSC-R10 is a cancer-specific instrument that allows the screening of cancer patients for the need of psycho-oncological treatment (Figure 1). This validated self-assessment questionnaire consists of 10 items, which were extracted from the QSC-R23 (3, 12). The 10 items of the QSC-R10 assess potentially stressful situations in the daily life of patients with cancer; the response categories are the same as for the QSC-R23 $(3,12)$. For each situation, patients indicate whether a statement currently applies to them and, if so, the severity of the problem. Response categories range between 0 (the problem does not apply to me) and 5 (it applies to me and is a very serious problem). The items are grouped into five subscales: Psychosomatic complaints, fears, information 


Here is a list of potentially stressful situations which might apply in your daily life. For each situ-
ation, please decide whether the situation applies to you. If so, please indicate how serious the
problem is for you by checking the box on the five-point scale "a slight problem" to "a very serious
problem". If not, then check the box "not applicable".
QSC - R10
1. I feel tired and weak.
2. I am in pain.
3. I feel physically imperfect.
4. I have too few opportunities to speak with a professional
about my psychological distress.
5. I am afraid that my disease will spread/recur.
6. It is difficult for my partner to empathize with my situation.
7. My sleep is disturbed.
8. It is harder for me to take part in recreation activities
(e.g. sports) now than it was before I became ill.
9. I do not feel well informed about my disease/treatment.
10. I feel tense and/or nervous.

Figure 1. Questionnaire on distress in cancer patients - short form (QSC-R10).

deficits, everyday life restrictions and social strains. The total score is calculated by summing the single-item scores. A total score of more than 14 points (the maximum possible score is 50 points) reveals a relevant level of psychosocial distress and indicates the need for psycho-oncological support (3).

In addition to the self-assessment questionnaire, patients are asked to complete two further questions:

i) "Do you want psychosocial support?" (response options: "Yes" and "No")

ii) "Do you currently receive psycho-oncological support?" (response options: "Yes" and "No")

Statistical analysis. Statistical analysis was performed with SPSS Statistics (IBM, Armonk, NY, USA). Descriptive statistics were calculated for sample characteristics. For each QSC-R10 item and for the total score, means and standard deviations were calculated. Data were statistically analysed according to the KolmogorovSmirnov test, and a standard distribution was assumed for all groups. To compare distress values, a further analysis was performed using multifactorial ANOVA and Bonferroni post hoc testing where applicable.

\section{Results}

Medical and demographic profile. In total, 202 patients [93 females (46\%) and 109 males (54\%)] met the inclusion criteria and were included in this study (Table I).
Psychosocial distress level. The distress score in our patients $(n=202)$ averaged 13.2 points (range $=0-42$ points; $S D=9.5)$; 72 patients $(36 \%)$ exceeded the cut-off value of 14 points in the QSC-R10 screening. The mean of single-item scores ranged from $0.64-2.18$ points $(\mathrm{SD}=1.07-1.55)$ (Table II). The highest mean score $(2.18$ points, $\mathrm{SD}=1.55)$ was recorded for item five ("I am afraid my disease will spread/recur") and the lowest (0.64 points, $\mathrm{SD}=1.07)$ for item nine ("I do not feel well informed about my disease/treatment").

The ANOVA revealed that female patients $(p=0.023)$ and patients who underwent radiotherapy $(p=0.021)$ had significantly higher distress scores.

There was no significant association between the variables of tumour grading $(p=0.140)$, tumour localization $(p=0.662)$, chemotherapy $(p=0.471)$, metastasis $(p=0.075)$ or duration of postoperative illness $(p=0.365)$ and high psychosocial distress. Patients with bone tumours had slightly higher distress levels, but this result did not reach statistical significance $(p=0.053)$.

Individual desire for psycho-oncological support and current (active) psycho-oncological treatment. In total, 32 patients (16\%) expressed an individual desire for psycho-oncological support; scores for 18 of those $(56 \%)$ exceeded the cut-off 
Table II. Stress scores for single items of the QSC-R10 (study sample, $n=202$ ).

\begin{tabular}{|c|c|c|c|}
\hline \multirow[b]{2}{*}{ Item } & & \multicolumn{2}{|c|}{ Score } \\
\hline & & Mean & $\mathrm{SD}$ \\
\hline 1 & I feel tired and weak. & 1.21 & 1.25 \\
\hline 2 & I have pain. & 1.45 & 1.44 \\
\hline 3 & I feel physically imperfect. & 1.22 & 1.40 \\
\hline 4 & I have too few opportunities to speak to a professional about my psychological distress. & 0.92 & 1.38 \\
\hline 5 & I am afraid that my disease will spread/recur. & 2.18 & 1.55 \\
\hline 6 & It is difficult for my partner to empathize with my situation. & 0.82 & 1.20 \\
\hline 7 & My sleep is disturbed. & 1.15 & 1.39 \\
\hline 8 & It is harder for me to take part in recreation activities (e.g. sports) now than it was before I became ill. & 2.15 & 1.80 \\
\hline 9 & I do not feel well informed about my disease/treatment. & 0.64 & 1.07 \\
\hline 10 & I feel tense and/or nervous. & 1.39 & 1.36 \\
\hline
\end{tabular}

value in the QSC-R10 screening. In the highly distressed cohort of patients $(\mathrm{n}=72), 18(25 \%)$ stated a desire for psycho-oncological support.

Twenty-six patients (16\%) were under psycho-oncological treatment at the time of the screening; scores for 15 of them (58\%) exceeded the cut-off value in the QSC-R10.

\section{Discussion}

The aim of this study was to gather preliminary data on the prevalence of psychosocial distress in sarcoma patients in follow-up care. Furthermore, we examined potential risk factors for psychosocial distress in patients with these rare tumours. We retrospectively enrolled 202 patients with bone or soft-tissue sarcomas, and they underwent a tablet-based cancer-specific distress screening during follow-up care at our Institution. More than one-third of patients were highly distressed at the time of the distress screening (mean of 31 months postoperatively) and received psycho-oncological treatment. Female patients and patients who underwent radiotherapy had significantly higher distress levels than males and patients without radiotherapy, respectively. Among patients with a postoperative illness duration of more than 2 years, approximately one-third were highly distressed. Our findings support the demand for psychosocial distress screenings not only during active treatment but also throughout follow-up care.

The percentage of distressed patients in our study was comparable to those in other studies that investigated psychosocial comorbidities in patients with cancer $(1,2)$. Zabora et al. analysed psychological distress in a large sample of 4,496 patients with 14 different cancer entities, and a distress prevalence of $35.1 \%$ was recorded (2). Similarly, in the study by Tang et al., approximately onethird of patients with extremity sarcomas were reported to suffer from psychosocial distress (18). However, most previous studies did not utilize cancer-specific distress questionnaires such as the QSC-R10. Herschbach et al., in contrast, assessed psychosocial distress in patients with cancer with a cancer-specific questionnaire (12). In a heterogeneous sample of 1,721 patients, participants with soft-tissue tumours accounted for the highest proportion of distressed patients, with $40 \%$ having scores exceeding the cut-off value and requiring psychosocial support. Pohlig et $a l$. conducted a social media-based cancer-specific survey in a young cohort of patients with bone sarcomas, and all enrolled patients had scores which exceeded the cut-off value (26). Due to the study design employed, a certain selection bias was assumed because distressed patients are probably more likely to participate in patient communities of social networks (26). Nevertheless, the extremely high percentage of psychological distress in paediatric sarcoma survivors has been described in the literature (19).

We believe that cancer-specific distress screenings help to mirror the subjective disease-related experiences of patients with cancer and, therefore, might be clinically relevant for determining the need for psycho-oncological support. The cancer-specific screening instrument used in this study (QSC-R10) was developed to assess potentially stressful situations in the daily life of patients with cancer. The most highly rated single distress item in our cohort was the fear of disease progression. Fear, in this context, is an adequate and logical response to the real threat of cancer. Approximately $50 \%$ of patients with cancer suffer from moderate to severe fear of disease progression (27). However, a high fear level can affect well-being, quality of life and social functioning and is, therefore, one of the most frequent distress symptoms in patients with cancer (28).

In line with previous studies, we found significantly higher levels of distress in women than in men $(2,10)$. Furthermore, we found that those patients who underwent radiotherapy were significantly more distressed than those 
who did not. One explanation might be the treatment setting, with daily radiation up to 8 weeks and possible side effects (e.g. radiodermatitis and wound-healing disorders). Several studies reported high distress levels and the need for psychooncological treatment during and after radiotherapy $(29,30)$. However, Herschbach et al. reported that for most cancer entities, radiation therapy did not play a major role in subjective stress perception (31).

In our cohort, the duration of postoperative illness did not have a statistically significant impact on overall distress levels. However, there was a tendency for patients with a postoperative illness duration of less than 6 months to have higher overall distress levels; scores for $47 \%$ of patients with an illness duration of less than 6 months exceeded the defined cut-off value, while those for $33 \%$ in the 6-12 month group and $35 \%$ in the $12-24$ month group exceeded the cutoff value. These findings are in line with those reported by Tang et al., who investigated the prevalence of psychosocial distress in 76 patients with extremity sarcomas at different time-points (preoperatively, 6 and 12 months postoperatively). The mean overall distress was highest after surgery and decreased over time, but similarly to our results, this difference did not reach statistical significance (18).

In our study, scores for $30 \%$ of patients with a postoperative illness duration of more than 2 years exceeded the cut-off value. Long-term distress was previously described in a systematic review by Foster et al., who reported that up to one-third of patients with cancer suffered from cancer-related problems, including poorer quality of life and psychosocial distress (32). The high number of patients with sarcoma with persistent psychosocial difficulties might be explained by the surgical treatment regimen, which, in some cases, involves restrictive or even mutilating operations (e.g. amputations) and might result in life-role changes as well as physical impairment $(33,34)$. In the cohort of highly distressed patients (those whose scores exceeded the cut-off value), only $25 \%$ expressed the desire to receive psycho-oncological support. A divergence between the objective indication (need) and the individual desire for psycho-oncological support has been described by de Zwaan et al., who concluded that treatment decisions should be based not only on the objective but also the self-reported need for help (35).

Our study has certain limitations. The study cohort was somewhat heterogeneous with regards to tumour entity (bone sarcomas and soft-tissue sarcomas), grading, and the timepoint of the survey in the course of cancer treatment. Furthermore, we did not consider the functional outcome, which might have had an impact on the psychosocial comorbidity of patients with cancer. The fact that only results of a single screening were included for each patient limits the significance of our study with regards to determination of time patterns in distress during follow-up care.

\section{Conclusion}

A large number of patients with bone and soft-tissue sarcomas suffer from psychosocial distress during follow-up care. In our cohort, marked psychosocial distress problems were observed in patients within the early postoperative phase ( $<6$ months), but approximately one-third of patients with a postoperative illness duration of more than 2 years also showed high psychosocial distress levels. Female gender and treatment with radiotherapy had significant negative impacts on the psychosocial distress level in our study.

The results underscore the importance of routine psychosocial distress screenings in patients with extremity sarcomas, which should be performed throughout the followup period.

\section{Conflicts of Interest}

All Authors declare that they have no conflict of interest in regard to this study.

\section{Authors' Contributions}

FL was responsible for data analysis/interpretation and article preparation. FP, CK, and HM were involved in the initial study idea, data analysis/interpretation and article preparation. HR, RvER, PH and TP were involved in the process of data interpretation and gave intellectual feedback. UL was one of the study investigators, was involved in data interpretation and in article preparation. All Authors read and approved the final version of the article.

\section{Acknowledgements}

This study was supported by the Wilhelm-Sander Foundation (project number: 2009.905.2), which is a charitable, non-profit foundation for the promotion of cancer research.

\section{References}

1 Mehnert A and Koch U: Psychological comorbidity and healthrelated quality of life and its association with awareness, utilization, and need for psychosocial support in a cancer register-based sample of long-term breast cancer survivors. J Psychosom Res 64(4): 383-391, 2008. PMID: 18374737. DOI: 10.1016/j.jpsychores.2007.12.005

2 Zabora J, BrintzenhofeSzoc K, Curbow B, Hooker C and Piantadosi S: The prevalence of psychological distress by cancer site. Psychooncology 10(1): 19-28, 2001. PMID: 11180574.

3 Book K, Marten-Mittag B, Henrich G, Dinkel A, Scheddel P, Sehlen S, Haimerl W, Schulte T, Britzelmeir I and Herschbach $\mathrm{P}$ : Distress screening in oncology-evaluation of the questionnaire on distress in cancer patients-short form (QSC-R10) in a German sample. Psychooncology 20(3): 287-293, 2011. PMID: 20669340. DOI: 10.1002/pon.1821

4 Mehnert A, Hartung TJ, Friedrich M, Vehling S, Brahler E, Harter M, Keller M, Schulz H, Wegscheider K, Weis J, Koch U and Faller H: One in two cancer patients is significantly distressed: 
Prevalence and indicators of distress. Psychooncology 27(1): 7582, 2018. PMID: 28568377. DOI:10.1002/pon.4464

5 Graves KD, Arnold SM, Love CL, Kirsh KL, Moore PG and Passik SD: Distress screening in a multidisciplinary lung cancer clinic: Prevalence and predictors of clinically significant distress. Lung Cancer 55(2): 215-224, 2007. PMID: 1857305. DOI: 10.1016/j.lungcan.2006.10.001

6 Siedentopf F, Marten-Mittag B, Utz-Billing I, Schoenegg W, Kentenich $\mathrm{H}$ and Dinkel A: Experiences with a specific screening instrument to identify psychosocial support needs in breast cancer patients. Eur J Obstet Gynecol Reprod Biol 148(2): 166-171, 2010. PMID: 19944516. DOI: 10.1016/j.ejogrb. 2009.10.014

7 Carlson LE and Bultz BD: Cancer distress screening. Needs, models, and methods. J Psychosom Res 55(5): 403-409, 2003. PMID: 14581094.

8 Mitchell AJ: Pooled results from 38 analyses of the accuracy of distress thermometer and other ultra-short methods of detecting cancer-related mood disorders. J Clin Oncol 25(29): 4670-4681, 2007. PMID: 17846453. DOI: 10.1200/JCO.2006.10.0438

9 Cape J and McCulloch Y: Patients' reasons for not presenting emotional problems in general practice consultations. Br J Gen Pract 49(448): 875-879, 1999. PMID: 1313556.

10 Singer S, Dieng S and Wesselmann S: Psycho-oncological care in certified cancer centres - a nationwide analysis in Germany. Psycho-oncology 22(6): 1435-1437, 2013. PMID: 22855347. DOI: $10.1002 /$ pon.3145

11 Grimer RJ and Briggs TW: Earlier diagnosis of bone and softtissue tumours. J Bone Joint Surg Br 92(11): 1489-1492, 2010. PMID: 21037340. DOI: 10.1302/0301-620X.92B11.24326

12 Herschbach P, Keller M, Knight L, Brandl T, Huber B, Henrich G and Marten-Mittag B: Psychological problems of cancer patients: A cancer distress screening with a cancer-specific questionnaire. $\mathrm{Br} \mathrm{J}$ Cancer 91(3): 504-511, 2004. PMID: 2409853. DOI: 10.1038/sj.bjc.6601986

13 Jacobs AJ, Michels R, Stein J and Levin AS: Improvement in overall survival from extremity soft tissue sarcoma over twenty years. Sarcoma 2015: 279601, 2015. PMID: 4363656. DOI: $10.1155 / 2015 / 279601$

14 Paredes T, Canavarro MC and Simoes MR: Anxiety and depression in sarcoma patients: Emotional adjustment and its determinants in the different phases of disease. Eur J Oncol Nurs 15(1): 73-79, 2011. PMID: 20667777. DOI: 10.1016/j.ejon. 2010.06.004

15 Trautmann F, Singer S and Schmitt J: Patients with soft-tissue sarcoma comprise a higher probability of comorbidities than cancer-free individuals. A secondary data analysis. Eur J Cancer Care 26(6), 2017. PMID: 27957779. DOI: 10.1111/ecc.12605

16 D'Agostino NM, Edelstein K, Zhang N, Recklitis CJ, Brinkman TM, Srivastava D, Leisenring WM, Robison LL, Armstrong GT and Krull KR: Comorbid symptoms of emotional distress in adult survivors of childhood cancer. Cancer 122(20): 3215-3224, 2016. PMID: 5048494. DOI: 10.1002/cncr.30171

17 Paredes T, Pereira M, Simoes MR and Canavarro MC: A longitudinal study on emotional adjustment of sarcoma patients: The determinant role of demographic, clinical and coping variables. Eur J Cancer Care 21(1): 41-51, 2012. PMID: 21812845. DOI: 10.1111/j.1365-2354.2011.01269.x

18 Tang MH, Castle DJ and Choong PF: Identifying the prevalence, trajectory, and determinants of psychological distress in extremity sarcoma. Sarcoma 2015: 745163, 2015. PMID: 4342175. DOI: $10.1155 / 2015 / 745163$
19 Wiener L, Battles H, Bernstein D, Long L, Derdak J, Mackall CL and Mansky PJ: Persistent psychological distress in longterm survivors of pediatric sarcoma: The experience at a single institution. Psychooncology 15(10): 898-910, 2006. PMID: 2289870. DOI: 10.1002/pon.1024

20 Pirl WF, Fann JR, Greer JA, Braun I, Deshields T, Fulcher C, Harvey E, Holland J, Kennedy V, Lazenby M, Wagner L, Underhill M, Walker DK, Zabora J, Zebrack B and Bardwell WA: Recommendations for the implementation of distress screening programs in cancer centers: Report from the American Psychosocial Oncology Society (APOS), Association of Oncology Social Work (AOSW), and Oncology Nursing Society (ONS) joint task force. Cancer 120(19): 2946-2954, 2014. PMID: 24798107. DOI: $10.1002 /$ cncr.28750

21 Snyder CF, Aaronson NK, Choucair AK, Elliott TE, Greenhalgh J, Halyard MY, Hess R, Miller DM, Reeve BB and Santana M: Implementing patient-reported outcomes assessment in clinical practice: A review of the options and considerations. Qual Life Res 21(8): 1305-1314, 2012. PMID: 22048932. DOI: 10.1007/ s11136-011-0054-x

22 Taenzer P, Bultz BD, Carlson LE, Speca M, DeGagne T, Olson $\mathrm{K}$, Doll R and Rosberger Z: Impact of computerized quality of life screening on physician behaviour and patient satisfaction in lung cancer outpatients. Psychooncology 9(3): 203-213, 2000. PMID: 10871716.

23 Koehler M HB, Holzner B, Schäffeler N, Zimmermann T, Nest A, Wifling $\mathrm{K}$ and Herschbach P: Future now - implementation of computer-assisted distress screening. Der Onkologe 23(6): 453-461, 2017. DOI: 10.1007/s00761-017-0209-7

24 Allenby A, Matthews J, Beresford J and McLachlan SA: The application of computer touch-screen technology in screening for psychosocial distress in an ambulatory oncology setting. Eur J Cancer Care 11(4): 245-253, 2002. PMID: 12492461.

25 Wright EP, Selby PJ, Crawford M, Gillibrand A, Johnston C, Perren TJ, Rush R, Smith A, Velikova G, Watson K, Gould A and Cull A: Feasibility and compliance of automated measurement of quality of life in oncology practice. J Clin Oncol 21(2): 374-382, 2003. PMID: 12525532. DOI: 10.1200/ JCO.2003.11.044

26 Pohlig F, Lenze U, Muhlhofer HML, Lenze FW, Schauwecker J, Knebel C, Zimmermann T and Herschbach P: IT-based psychosocial distress screening in patients with sarcoma and parental caregivers via disease-specific online social media communities. In Vivo 31(3): 443-450, 2017. PMID: 5461459. DOI: 10.21873 /invivo.11081

27 Herschbach P and Dinkel A: Fear of progression. Recent Results Cancer Res 197: 11-29, 2014. PMID: 24305766. DOI: 10.1007/978-3-642-40187-9_2

28 Dinkel A and Herschbach P: Fear of progression in cancer patients and survivors. Recent Results Cancer Res 210: 1333, 2018. PMID: 28924677. DOI: 10.1007/978-3-319-64310$6 \_2$

29 Chen AM, Jennelle RL, Grady V, Tovar A, Bowen K, Simonin P, Tracy J, McCrudden D, Stella JR and Vijayakumar S: Prospective study of psychosocial distress among patients undergoing radiotherapy for head and neck cancer. Int J Radiat Oncol Biol Phys 73(1): 187-193, 2009. PMID: 18513884. DOI: 10.1016/j.ijrobp.2008.04.010

30 Sehlen S, Hollenhorst H, Schymura B, Herschbach P, Aydemir U, Firsching $M$ and Duhmke E: Psychosocial stress in cancer 
patients during and after radiotherapy. Strahlenther Onkol 179(3): 175-180, 2003. PMID: 12627260. DOI: 10.1007/s00066003-1018-z

31 Herschbach P, Book K, Brandl T, Keller M, Lindena G, Neuwohner K and Marten-Mittag B: Psychological distress in cancer patients assessed with an expert rating scale. Br J Cancer 99(1): 37-43, 2008. PMID: 2453012. DOI: 10.1038/sj.bjc. 6604420

32 Foster C, Wright D, Hill H, Hopkinson J and Roffe L: Psychosocial implications of living 5 years or more following a cancer diagnosis: A systematic review of the research evidence. Eur J Cancer Care 18(3): 223-247, 2009. PMID: 19432917. DOI: $10.1111 / \mathrm{j} .1365-2354.2008 .01001 . x$

33 Griesser MJ, Gillette B, Crist M, Pan X, Muscarella P, Scharschmidt $\mathrm{T}$ and Mayerson $\mathrm{J}$ : Internal and external hemipelvectomy or flail hip in patients with sarcomas: Qualityof-life and functional outcomes. Am J Phys Med Rehabil 91(1): 24-32, 2012. PMID: 22042339. DOI: 10.1097/PHM.0b $013 \mathrm{e} 318232885 \mathrm{a}$
34 Mason GE, Aung L, Gall S, Meyers PA, Butler R, Krug S, Kim M, Healey $\mathrm{JH}$ and Gorlick R: Quality of life following amputation or limb preservation in patients with lower extremity bone sarcoma. Front Oncol 3: 210, 2013. PMID: 3742996. DOI: 10.3389/fonc. 2013.00210

35 de Zwaan M, Mosch P, Sinzinger H, Stresing K, Oberhof P, Kohl C, Schilke C and Muller A: The association between the need for psychosocial support, patients' desire for psychosocial support and received psychosocial interventions in cancer patients. Neuropsychiatr 26(4): 152-158, 2012. PMID: 23179358. DOI:10.1007/s40211-012-0035-5

Received April 28, 2019

Revised May 10, 2019

Accepted May 13, 2019 